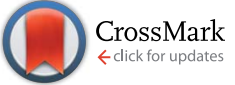

Cite this: RSC Adv., 2016, 6, 70414

\title{
Seed mediated growth of silver nanoplates on glass: exploiting the bimodal antibacterial effect by near IR photo-thermal action and $\mathrm{Ag}^{+}$release $\uparrow$
}

\author{
A. D'Agostino, ${ }^{a}$ A. Taglietti, ${ }^{\star a}$ P. Grisoli, ${ }^{b}$ G. Dacarro, ${ }^{C}$ L. Cucca, ${ }^{a}$ M. Patrini ${ }^{c}$ \\ and P. Pallavicini ${ }^{a}$
}

\begin{abstract}
We developed a reproducible synthetic method to grow anisotropic silver nanoplates on glass, giving them the desired plasmonic features and tuning their size and LSPR absorption with growth time. Samples were fully characterized showing good stability, a strong photo-thermal effect and the ability to release controlled quantities of silver in water. These samples showed a strong antibacterial activity, which is based on two different mechanisms: silver ion release and hyperthermia caused by the photo-thermal effect under near-infrared laser irradiation. This behaviour allows us to foresee promising in vivo applications, ensuring a long-term antibacterial protection which can be reinforced, when needed, by a fast photo-thermal action which can be switched on by a NIR laser treatment.
\end{abstract}

Received 4th May 2016

Accepted 19th July 2016

DOI: 10.1039/c6ra11608f

www.rsc.org/advances

action involving the direct interaction of AgNPs with the bacterial cell membrane. ${ }^{3}$ In the last years we confirmed that AgNPs are effective towards Gram- bacterial strains but much less towards $\mathrm{Gram}+{ }^{4}{ }^{4}$ and that this action can be easily brought on a bulk glass surface by means of interactions of AgNPs surfaces with a -SH layer, after forming first a layer of mercaptopropyltriethoxy silane (MPTS) ${ }^{3,5}$ Similar results were obtained attaching a monolayer of AgNPs on a layer of polyethyleneimine (PEI) polymer, a strategy which allows to establish electrostatic interactions between its amino groups and AgNPs surfaces. ${ }^{6}$ Recently we also demonstrated that a monolayer of AgNPs obtained through preliminary silanization of the glass with 3-aminopropyltriethoxysilane (APTES) can be conveniently used against the biofilm-producer Staphylococcus epidermidis RP62A. $^{7}$ In all these cases, excellent stability in aqueous media, prolonged release of silver ions and high local concentration of $\mathrm{Ag}^{+}$are obtained, without any detaching of AgNPs and resulting in a strong antibacterial action with limited risks towards human health. Another way to use noble metal nanoparticles to fight bacteria presence and growth is to exploit their photo-thermal features: ${ }^{8}$ for example, gold nanorods (GNRs) ${ }^{9}$ and gold nanostars (GNSs) ${ }^{10}$ have been used to destroy colonies of different bacterial strains. Gold is not intrinsically antibacterial, anyway anisotropic gold nano-objects featuring two or more localized surface plasmon resonances (LSPR) that undergo thermal relaxation when irradiated, can produce a localized hyperthermia which is used to kill bacteria and eradicate biofilms. Moreover, when at least one LSPR falls in the near-IR range (NIR, 750-950 $\mathrm{nm}$ ) where tissues and blood are semi-transparent, one can imagine to use laser irradiation on subcutaneous devices coated with these kind of nanomaterials. In a recent example, ${ }^{11}$ we prepared active surfaces 
bearing controlled microgram quantities of gold as grafted monolayers of GNS. Laser irradiation of the GNS monolayer on their NIR LSPR results in efficient photo-thermal conversion, and we observed that the produced hyperthermia efficiently induced bacteria cell death in $S$. aureus biofilms. Photo-thermal effects have also been be exploited to increase silver ions release from bimetallic nanostructures, and then considering this release for microbicidal tasks. In a recent example it was found that Pd@Ag nanosheets show a synergistic effect between silver ions and photo-thermal heating obtained from the plasmonic objects produced reducing silver on Pd nanosheet surfaces: upon irradiation in the NIR region, the hyperthermia caused an increase of silver ions release, resulting in an enhanced antibacterial action of the colloidal nanomaterial. ${ }^{12}$ Other examples are reported of combined action between silver bactericidal action and gold photo-thermal features, obtained upon irradiation in the visible region in the case of gold core/silver shell spherical nanoparticles stabilized with aspartame, ${ }^{13}$ or in the NIR in the case of gold core/silver shell nanorods. ${ }^{14}$ Curiously, few examples are reported about the photo-thermal action of colloidal suspensions of nano-objects made of pure silver. The ability to give an efficient photo-to-heat conversion, in fact, is not only limited to gold and palladium nano-objects: silver itself can be used as well. Recent and pioneering reports from Astilean and coworkers ${ }^{15,16}$ fully exploit this feature, showing the successful use of biocompatible chitosan-coated silver nanoplates for photo-thermal treatments of cancer cells when irradiated at $800 \mathrm{~nm}$, a wavelength value where a plasmonic band can be easily placed by tuning morphology and dimensions of nanoplates themselves. It is well known that an LSPR band in the NIR can be obtained using anisotropic silver objects of different morphologies, exploiting the in plane (longitudinal) dipolar resonance. ${ }^{17}$ To our knowledge, anyway, no examples of antibacterial action obtained with hyperthermia coming from pure silver plasmonic structures is reported, nor in colloidal suspension neither grafted to a surface. Seed-growth methods represent a very popular way to prepare anisotropic noble metal nano-objects, and are often based on the use of surfactant molecules to orient the anisotropic growth. ${ }^{18,19}$ Unfortunately, it can be hard to remove them from the nanoparticles surfaces, thus complicating further chemistry which could be needed for further functionalizations. ${ }^{20}$ Also, surfactants may have an harmful effect when used in situations connected to health, as they are in some cases considered toxic for human cells. ${ }^{21}$ Seedgrowth methods can be extended to grow anisotropic nanoobjects directly on surfaces: a few examples, mainly in presence of surfactants, of seed-growth synthesis of silver anisotropic nano-objects on different kinds of substrates have been reported. ${ }^{20,22-26}$ In a recent example, a seed mediated growth of silver nanoplates on a glass surface was obtained, with a certain degree of control, through several successive immersion steps, using citrate as orienting agent allowing only their longitudinal growth, and obtaining the generation of a very broad absorption covering the whole vis-NIR spectra. ${ }^{27}$

Here, we demonstrate how to prepare bulk glass materials bearing silver nanoplates, tailored to have an high absorption in the NIR region and having strong photo-thermal features, in order to study their potential as model antibacterial surfaces. The long-term goal is the possibility of fabricating medical devices with coatings which could be active on long timescales as a result of the "classical" antibacterial effect due to silver ions release, ${ }^{3-7}$ but with a further, additional antibacterial action which could be "switched on" on need by a NIR laser irradiation, producing localized hyperthermia, ${ }^{12}$ and to evaluate the possible synergy between these two kind of actions. In order to minimize the use of possibly toxic chemicals and avoid the use of classical surfactants, we introduce here a surfactant free seed-growth method to be applied directly on surfaces, involving the use of citrate as a shape directing agent. The idea was to tune the geometric features of the nano-objects in a single growing step maintaining an easy synthetic setup and just controlling the growth time. In this way an easy control the LSPR features of the samples is obtained, and an intense absorption in the NIR region can be gained and exploited for efficient laser induced photo-thermal effect.

\section{Experimental}

\section{Materials}

Silver nitrate ( $>99.8 \%$ ), sodium borohydride $(>99.0 \%)$, sodium citrate $(>99.0 \%)$, ascorbic acid $(\geq 99 \%)$ were purchased from Sigma Aldrich. Trimethoxysilylpropyl(polyethylenimine) (50\% in isopropanol) was purchased from Gelest Inc. Reagents were used as received. Solvents were purchased from Sigma-Aldrich and used as supplied. Microscopy cover glass slides $(21 \times$ $26 \mathrm{~mm}$ ) were purchased from DelChimica. Water was deionized and then bidistilled.

Tryptone Soya Broth (TSB) and Tryptone Soya Agar (TSA) for bacteria culture were purchased from Oxoid, England. S. aureus ATCC 6538 and E. coli ATCC 10536 bacterial strains were used.

\section{Preparation of PEI-silane self-assembled monolayers on glass surface (SURF-PEI glasses)}

The samples were prepared according a reported method. ${ }^{6}$ Briefly: glass substrates were cleaned for $30 \mathrm{~min}$ in freshly prepared Piranha solution $\left(3: 1 \mathrm{v} / \mathrm{v} \quad \mathrm{H}_{2} \mathrm{SO}_{4}: \mathrm{H}_{2} \mathrm{O}_{2} \quad(30 \%)\right)$. Caution! Piranha solution is a strong oxidizing agent and should be handled with care.), washed three times with ultrapure water in a sonic bath and oven-dried. The slides were then immersed for $6 \mathrm{~min}$ in a $4 \%(\mathrm{v} / \mathrm{v})$ solution of PEI-silane in ethanol at room temperature. In a typical preparation, 8 glass slides were prepared at the same time, i.e. reacting in the same silane solution inside a 8-place holder (a microscope glass slides staining Jar). After this, the slides were washed two times with ethanol and one time with ultrapure water in a sonic bath and blow-dried with nitrogen.

\section{Seeds preparation}

Seeds, spherical silver nanoparticles, were prepared according to a reported method. ${ }^{5}$ Briefly, to $100 \mathrm{~mL}$ of ice cooled water the following solutions were added in sequence under vigorous stirring $1 \mathrm{~mL}$ of $1 \%(\mathrm{w} / \mathrm{v}) \mathrm{AgNO}_{3}$ solution, after a minute $1 \mathrm{~mL}$ of $1 \%(\mathrm{w} / \mathrm{v})$ sodium citrate and, after a further minute, $0.500 \mathrm{~mL}$ of 
an ice-cooled solution $0.075 \mathrm{wt} \%$ in $\mathrm{NaBH}_{4}$ and $1 \mathrm{wt} \%$ in sodium citrate. After the last addition, stirring was immediately stopped, in order to avoid coagulation. The colloidal suspensions were stored in the preparation flask, maintained in the dark and used within 2 days from preparation.

\section{Preparation of SURF-PEI-SEEDS samples}

The samples were prepared according a reported method. ${ }^{6}$ PEI glasses were immersed in the colloidal suspension of seeds at room temperature for $15 \mathrm{~min}$. In a typical preparation, 8 glass slides were prepared at the same time, i.e. they were made to react in the same seeds suspension $(30 \mathrm{~mL})$, inside a glass holder (Hellendhal staining Jars were used) where the slides were kept in vertical position. After this the obtained glass samples, showing a yellow colour, were placed in water and sonicated for $5 \mathrm{~min}$. This procedure was repeated twice and then the glasses were blow dried with a nitrogen stream.

\section{Growth solution preparation}

Growth solution was prepared in a flask: to $30 \mathrm{~mL}$ of sodium citrate $8.5 \times 10^{-3} \mathrm{M}$ were added, under vigorous stirring, $18 \mathrm{~mL}$ of $\mathrm{AgNO}_{3} 5 \times 10^{-4} \mathrm{M}$ and $0.450 \mathrm{~mL}$ of ascorbic acid $1 \times 10^{-2} \mathrm{M}$. After the last addition, stirring was immediately stopped.

\section{Preparation of SURF-PEI-PLATES samples}

In a typical preparation, up to 8 SURF-PEI-SEEDS samples were immersed in the proper amount of growth solution for the desired time in a Hellendhal staining Jar. Changing in colour of sample was observed from yellow, characteristic colour of SURFPEI-SEEDS glass, to orange, red and dark blue on increasing dipping times. After this, the slides were washed with bidistilled water two times and dried under nitrogen flux.

\section{Antibacterial activity tests}

The antibacterial activity of functionalized cover glasses was investigated against Staphylococcus aureus ATCC 6538 (Gram+) and Escherichia coli ATCC 10356 (Gram-). The microorganisms were grown overnight in Tryptone Soya Broth (Oxoid; Basingstoke, Hampshire, England) at $37{ }^{\circ} \mathrm{C}$. Washed cells were resuspended in Dulbecco's PBS and optical density (OD) was adjusted to 0.2 at $650 \mathrm{~nm}$ wavelength, corresponding approximately to $1 \times 10^{8}$ Colony Forming Units (CFU) per mL. $10 \mu \mathrm{L}$ of bacterial suspension was deposited on a standard glass slide (76 $\times 26 \mathrm{~mm}$ ), then the microbial suspension was covered with a functionalized cover glass slide $(21 \times 26 \mathrm{~mm})$, forming a thin film between the slides that facilitates direct contact of the microorganisms with the active surface. The two assembled glasses were introduced in a Falcon test-tube $(50 \mathrm{~mL})$ containing $1 \mathrm{~mL}$ of PBS to maintain a damp environment. In this test microbes are incubated in non-nutritive suspensions that do not give the microorganisms the potential to grow during the test. For each bacterial strain three equivalent modified glasses were prepared; the slides were maintained in contact with the liquid films containing bacteria at room temperature for 20 minutes, 5 and $24 \mathrm{~h}$, respectively; for each time of contact an unmodified glass slide was treated in the same way as control sample. After the times of contact, $9 \mathrm{~mL}$ of PBS were introduced in each Falcon test-tube under a gentle shaking to detach the assembled glass slides. Bacterial suspensions were then cultured on Tryptone Soya Agar (Oxoid; Basingstoke, Hampshire, England) to count viable cells. The decimal-log reduction rate, i.e. the Microbicidal Effect (ME), was calculated using the formula:

$$
\mathrm{ME}=\log N_{\mathrm{C}}-\log N_{\mathrm{E}}
$$

( $N_{\mathrm{C}}$ being the number of $\mathrm{CFU} \mathrm{mL}^{-1}$ developed on the unmodified control glasses, and $N_{\mathrm{E}}$ being the number of $\mathrm{CFU} \mathrm{mL} \mathrm{m}^{-1}$ counted after exposure to modified glasses). The results expressed as ME represent the average of three equivalent determinations. This method can be considered as a version of the Japanese Industrial Standard JIS Z 2811 developed to measure the antibacterial activity of plastic surfaces.

\section{Thermal microbicidal tests}

Antibacterial activity due to photo-thermal effect of SURF-PEIPLATES was investigated against Staphylococcus aureus ATCC 6538 (Gram+) and Escherichia coli ATCC 10356 (Gram-). One functionalized slide was cut in 4 sections of $10 \times 10 \mathrm{~mm}$ in order to be completely irradiated by laser. A volume of $0.02 \mathrm{~mL}$ of bacterial suspension was deposited on 2 sections. For each pair of functionalized glasses, one was irradiated for $20 \mathrm{~min}$ whereas the other was not irradiated. After this time, the glass section covered with bacterial suspensions was suspended in 1 $\mathrm{mL}$ of sterile water, gently shaken and then water was suitable diluted in 3 different tubes: 1 : 100, $1: 10$ 000, $1: 100$ 000. From each tube, $1 \mathrm{~mL}$ of suspension was taken and then cultured on Tryptone Soya Agar (Oxoid; Basingstoke, Hampshire, England) to count viable cells. The decimal-log reduction rate, i.e. the "thermal microbicidal effect", $\mathrm{ME}_{\mathrm{T}}$ was calculated with the following formula

$$
\mathrm{ME}_{\mathrm{T}}=\log N_{\mathrm{C}}-\log N_{\mathrm{T}}
$$

where $N_{\mathrm{C}}$ is the number of CFU mL ${ }^{-1}$ developed in contact with a not irradiated modified control glass sample, and $N_{\mathrm{T}}$ the number of $\mathrm{CFU} \mathrm{mL} \mathrm{m}^{-1}$ counted after exposure to modified glass samples and irradiation.

\section{Instrumentation and instrumental methods}

Absorbance spectra of colloidal suspensions were taken with a Varian Cary 100 spectrophotometer in the $200-900 \mathrm{~nm}$ range and spectra of functionalized glasses were obtained placing the slides on the Varian Cary 100 spectrophotometer equipped with a dedicated Varian solid sample holder.

SEM images were taken from Tescan Mira XMU variable pressure Field Emission Scanning Electron Microscope - FEG SEM (Tescan USA Inc., USA) located at the Arvedi Laboratory, CISRiC, Pavia. Slides were mounted onto aluminum stubs using double sided carbon adhesive tape and were then made electrically conductive by coating in vacuum with a thin layer of $\mathrm{Pt} /$ Pd (3-5 nm). Observations were made in backscattered 
electrons mode (BSE) at $30 \mathrm{kV}$ and with InBeam secondary electron detector for higher spatial resolution. Textural observations, together with major element compositions, were performed by means of a Tescan FE-SEM (Mira 3XMU-series), equipped with an EDAX energy-dispersive spectrometer. The operating conditions were: $20 \mathrm{kV}$ accelerating voltage, around $40 \mathrm{~mA}$ beam current, $15.8 \mathrm{~mm}$ working distance, counts of $100 \mathrm{~s}$ per analysis. The measurements were processed using the EDAX Genesis software and semiquantitative data obtained using the ZAF correction.

Thermograms (temperature $v s$. time) were recorded on SURF-PEI-PLATES samples by means of a laser (multimode AlGaAs laser diode, L808P200, Thorlabs GmbH, emitting light at the wavelength of about $808 \mathrm{~nm}$, power of radiation is 200 $\mathrm{mW}$, irradiance is $0.26 \mathrm{~W} \mathrm{~cm}^{-2}$ ). Temperature was recorded by means of a FLIR E40 thermocamera and FLIR Tools+ software.

X-ray powder diffraction measurements were performed by using a Bruker D5005 diffractometer with the CuK $\alpha$ radiation, graphite monochromator and scintillation detector. The patterns were collected with a step size of $0.03^{\circ}$ and counting time of $2 \mathrm{~s}$ per step in the angular range $20-65^{\circ}$.

The total Ag content of SURF-PEI-PLATES was determined by quantitatively oxidizing the silver plates grafted on a single slide by dipping it in $3 \mathrm{~mL}$ ultrapure concentrated $\mathrm{HNO}_{3}$ diluted 1 : 5 with water in a becker, and keeping it overnight at RT on a Heidolph Promax 1020 reciprocating platform shaker. The Ag content in solution was then determined by inductively coupled plasma optical emission spectroscopy (ICP-OES) with an ICPOES OPTIMA 3300 DV Perkin Elmer instrument. The measure was repeated on 8 glass samples coming from 8 different preparations.

Release of $\mathrm{Ag}^{+}$versus time was followed on a set of 10 SURFPEI-PLATES slides $(21 \times 26 \mathrm{~mm}$ coated on both sides, total coated surface $10.92 \mathrm{~cm}^{2}$ ) prepared as described above. Each slide was then immersed in $3 \mathrm{~mL}$ of ultrapure water. Slides were taken off the water after 20 minutes, 1 h, 3 h, 5 h, 24 h, 48 h, 96 $\mathrm{h}, 130 \mathrm{~h}, 168 \mathrm{~h}$ and $240 \mathrm{~h}$. A Uv-vis spectra was measured on the water solution, and then the content of $\mathrm{Ag}^{+}$was determined by ICP-OES. Measures were repeated three times, and mean values are given. ICP data were collected with an ICP-OES OPTIMA 3300 DV Perkin Elmer instrument.

Release of $\mathrm{Ag}^{+}$of SURF-PEI-PLATES after laser excitation of 20 minutes was determined in the following mode. A $21 \times 26$ $\mathrm{mm}$ sample was cut in 4 sections of approximately $10 \times 10 \mathrm{~mm}$, with a surface which could be almost completely covered by the $1 \mathrm{~cm}^{2}$ laser spot. The four samples were placed in four wells and covered with $0.75 \mathrm{~mL}$ of bidistilled water and each one was irradiated for $20 \mathrm{~min}$ with a $808 \mathrm{~nm}$ laser source. After irradiation time the water fractions were reunited obtaining $3 \mathrm{~mL}$ of water. A UV-vis spectrum was measured and then the content of $\mathrm{Ag}^{+}$was determined by ICP-OES.

\section{Results and discussion}

\section{Seed growth synthesis}

As a first step of the synthetic strategy outlined in Scheme 1, we prepared a surface ready to graft small AgNPs to be used as

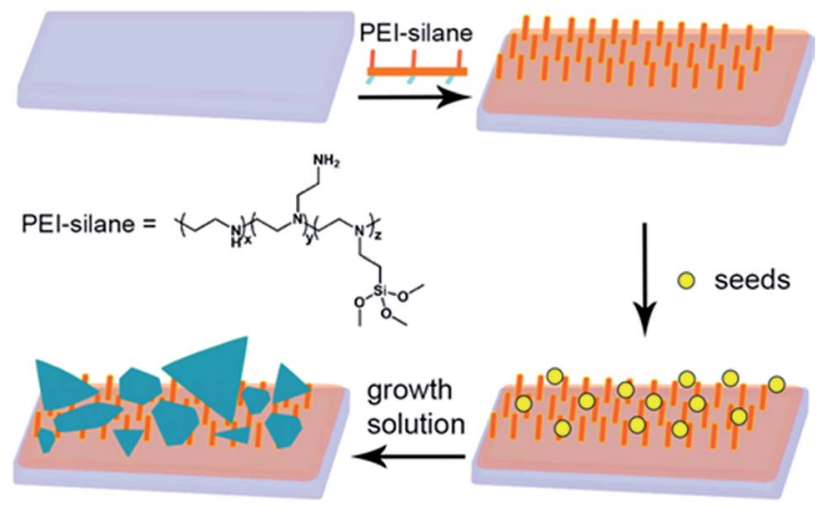

Scheme 1 Seed mediated growth of Ag nanoplates on PEI functionalized glass substrates plates.

seeds for the growth of anisotropic silver objects. We can prepare such a surface using PEI-silane (see Scheme 1), a polymer composed of an average of 3.6 propyltrimethoxysilane groups and 56 ethylenimine monomers, i.e. 56 amine moieties (including primary, secondary and tertiary amines). Each polymer molecule has multiple alkoxysilane attaching points, so it is possible to graft polymer molecules on silica surfaces by an easy and quick procedure, producing a monolayer. In the standard procedure, glass slides were cleaned and activated with a strong acidic and oxidizing solution, producing a completely hydrophilic surface (contact angle $<10^{\circ}$ ) which reacts in a few minutes with an ethanolic solution of PEI-silane.

After this step, PEI-functionalized surfaces showed a static contact angle of $41 \pm 5^{\circ}$ on glass, in good agreement with our previous results. Grafting of AgNPs was obtained by dipping the glass slides bearing SURF-PEI for 15 minutes in aqueous colloidal suspensions of citrate capped AgNPs, prepared according to a standard method which gives objects having a diameter of $7 \pm 4 \mathrm{~nm}$. Dipping time was kept to 15 minutes to avoid uncontrollable growth observed for longer immersion times, probably due to aggregation on surface, as we observed earlier. The typical spectra of a sample shows the presence of a LSPR band centred at 403 clearly indicating the presence of small AgNPs grafted on glass (see Fig. S1 in ESI $\dagger$ ). Full characterization of these samples has already been reported. ${ }^{6}$ As a control, total Ag quantity grafted on glasses was measured using of ICP-OES, after quantitative oxidation of NP on functionalized glasses and analyzing the obtained solutions, obtaining a concentration of $7.4( \pm 1.3) \times 10^{-7} \mathrm{~g} \mathrm{~cm}^{-2}$ of $\mathrm{Ag}$ as previously reported. ${ }^{6}$ A SEM image, indicating the homogeneous coating of the SURF-PEI glass samples with AgNPs (SURFPEI-SEEDS samples) is reported in the ESI part (see Fig. S2 $\dagger$ ).

Growth of the seeds grafted on surface was obtained placing the SURF-PEI-SEEDS glass samples in a growth chamber containing the growth solution. The growth solution was prepared in water adding $\mathrm{AgNO}_{3}$ as silver source, ascorbic acid as reducing agent and citrate as shape-directing agent. As already mentioned, citrate anions preferentially bind to 111 facets of the crystals which are forming, slowing down the vertical expansion, and thus allowing to obtain anisotropic growth of 
the objects to give nanoplates. ${ }^{27}$ These flat nano-objects typically show an in-plane dipole plasmon resonance band which position can be placed in the NIR region and tuned at a desired value just by regulating the aspect ratio of the objects. ${ }^{28,29}$ Our expectations were that it was possible to obtain increasing anisotropic growth on surface just increasing immersion time in presence of high citrate concentration, so that control of immersion times could allow tuning of the morphology and consequently the LSPR features of plasmonic objects brought on glass samples.

Fig. 1 shows the UV-Vis-NIR spectra measured for samples extracted from the growth chamber at different interval times. The development of a broad, intense band which maximum, in about two hours, progressively shifts to NIR values, can be attributed to the growth of objects having different anisotropic shapes and increasing lateral dimensions.

When the experiment was repeated, in the same growth solution, on simple SURF-PEI samples, i.e. in absence of grafted seeds, no objects formation and growth was observed, yielding transparent and uncoated glass samples. It is important to stress one of the main results coming from this experiment: with this method the maximum of LSPR spectra of the obtained samples can be tuned to the desired value, in the range between 400 and $800 \mathrm{~nm}$, just varying the immersion time of the seeds coated samples in the growth solution.

Increase of immersion times longer than two hours does not produce any noticeable change in spectra, as can be clearly seen in Fig. 1 (green dashed line spectrum) indicating that no further growth or shape changes are happening after this growing time.

SEM images were taken for the samples extracted after different immersion/growing times, and are reported in Fig. 2. Growth of objects can be immediately perceived moving from Fig. 2a (sample after $15 \mathrm{~min}$ immersion) to Fig. 2b (30 $\mathrm{min}$ immersion) to Fig. 2c (2 hours immersion).

In Fig. 2d, reporting a SEM image at an higher magnification, which is again relative to the two hours immersion time, the anisotropic growth of objects is evident: triangular and truncated triangular plates, with longitudinal dimensions

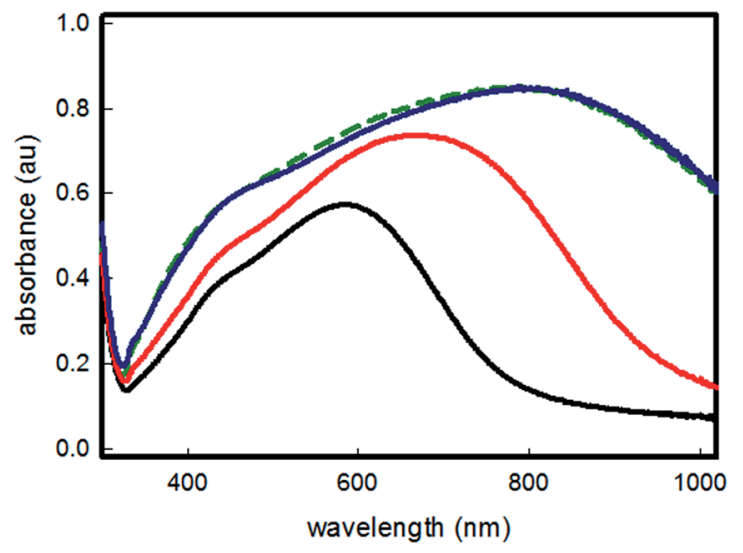

Fig. 1 UV-Vis-NIR spectra of samples after different times of seed mediated growth: 15 minutes (black line), 30 minutes (red line), two hours (blue line), 4 hours (green dashed line).
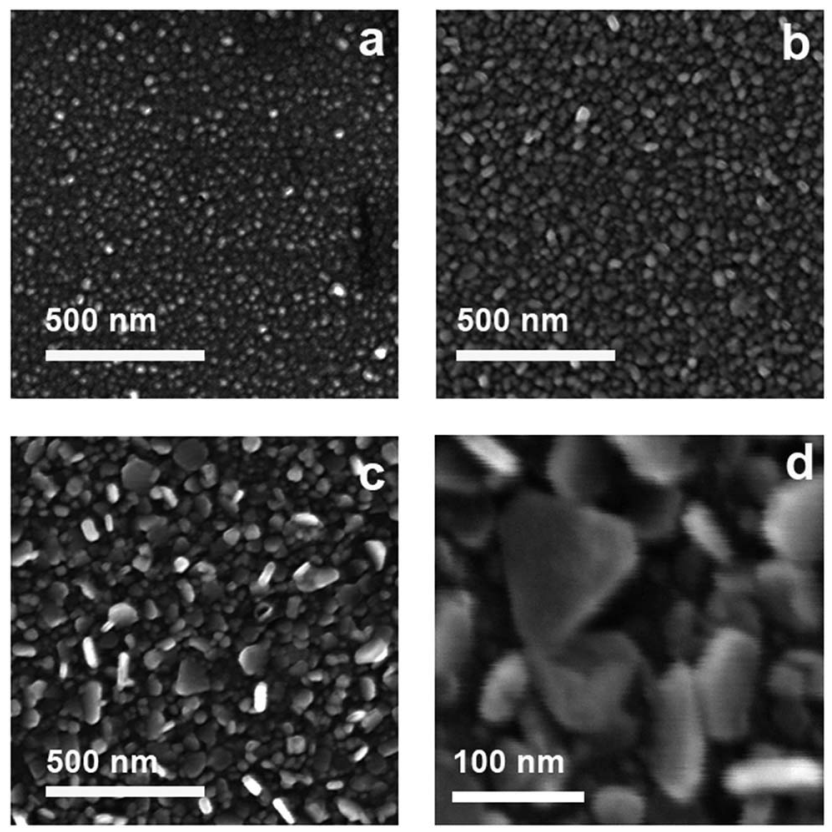

Fig. 2 SEM images of samples after different times of seed mediated growth: (a) 15 minutes, (b) 30 minutes, (c) 2 hours, (d) 2 hours, higher magnification.

ranging between 40 and $120 \mathrm{~nm}$ can be observed, all having a thickness not exceeding a value of $20 \mathrm{~nm}$. The extremely high degree of poly-dispersion explains the very broad LSPR spectra observed for increasing growth time. Nevertheless, the NIR absorption spectra is explainable with the presence of a distribution of objects having different longitudinal dimensions, and a subsequent broad range of dipolar in-plane resonance wavelength. ${ }^{25-29}$ The glass samples have an homogeneous dark bluegrey colour, which is consistent with the poly-dispersed array of grafted silver nanoplates and the LSPR features described: a photograph of a representative slide after two hours growth is reported in ESI $\dagger$ section.

As with two hours immersion the broad LSPR band was placed close to the desired wavelength value, we decided to use this growth time in the standard preparation for SURF-PEIPLATES samples. The preparation of SURF-PEI-PLATES is reproducible: spectra of several different samples are given in Fig. S3 and S4 in the ESI $\uparrow$ section, and evidence that no sensible differences in intensity and position of the LSPR peak can be observed among the samples obtained simultaneously from the same preparation (Fig. S3†) or coming from different preparation batches (Fig. S4†). It also ensures a very homogeneous coating of the surface, as can be seen from the SEM images taken on large area (see Fig. S5 in ESI $\dagger$ section). SURF-PEIPLATES slides are stable in air, i.e. their spectra and colour did not change significantly for at least a 4-week period.

EDX analysis (see ESI $\dagger$ ) were performed. Beyond the glass substrate components, the evidence of $\mathrm{Ag} \mathrm{L}$ spectral line is consistent with the preparation procedure. We notice that EDX analysis in different points of the sample surface shows chemical homogeneity of $\mathrm{Ag}$ films (within $\pm 5 \%$ ), the intensity of any spectral line in EDX analysis being proportional to element 
abundance. The presence of Ag plates is confirmed also from Xray diffraction patterns (see ESI $\dagger$ ). Only the peak of 111 reflection of a FCC structure is clearly evident, suggesting that the basal lattice plane of nano-objects is (111), as expected from the shape-directing action of citrate, and also that exists a preferred orientation of plates on the glass surface. The peak broadening confirms the nanostructured nature of silver.

We completed the characterization of the SURF-PEI-PLATES (samples c) by means of quantitative oxidation of the silver plates, and analysis of the obtained $\mathrm{Ag}^{+}$solution by means of ICP. Data from eight experiments, coming from eight different samples preparations, we found an average of $6.2(0.6) \times 10^{-6} \mathrm{~g}$ $\mathrm{cm}^{-2}$ of silver, a quantity which is considerably high, when compared to samples obtained grafting a monolayer of spherical seeds on a silanized glass, as we have described in the recent past. ${ }^{5-7}$

\section{Stability in water and silver release}

The stability in water, as well their ability to release silver ions in water as a function of time was determined by experiments on several SURF-PEI-PLATES samples (see experimental part). The $21 \times 26 \mathrm{~mm}$ glass slides were immersed in $3 \mathrm{~mL}$ of ultrapure water for a given time interval, and then removed from the liquid. After registering their UV-Vis-NiR spectra, the solutions obtained after SURF-PEI-PLATES slides immersion in water were analysed with ICP. The data of silver concentration found in these solutions were expressed as $\mu \mathrm{g}$ of released silver per $\mathrm{cm}^{2}$ of exposed surface, as a function of time (see Fig. 3), showing a well-defined trend. We must stress the fact that, in analogy with our precedent investigations, these experiments were performed in ultrapure water. ${ }^{5-7}$ Thus, the results cannot be of course simply transferred to more complex or biological environments, as silver ions can give poorly soluble salts or complexation with biomolecules when they are released in "real" media. ${ }^{30}$

Nevertheless these experiments allow to state that (i) no silver nano-objects were released in water, as Uv-Vis-NIR spectra did not show the presence of any absorption which could be related to presence of plasmonic objects detached from the

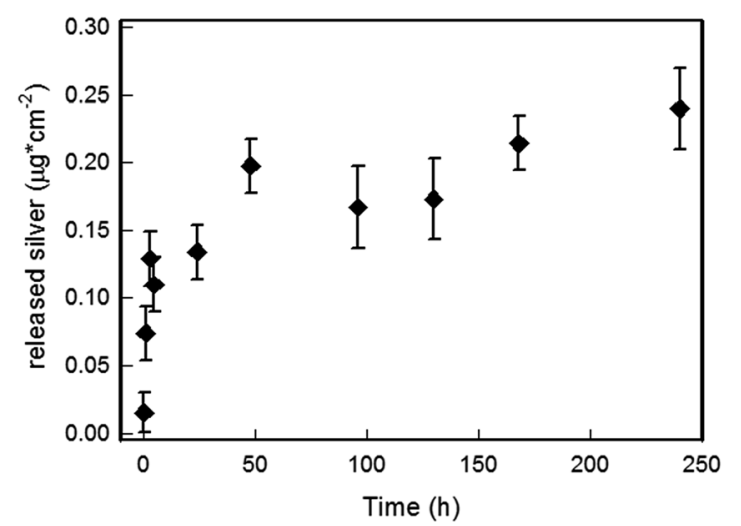

Fig. 3 Silver ion released from SURF-PEI-PLATES $\left(\mu \mathrm{g} \mathrm{cm}^{-2}\right.$ of exposed surface) versus time in $3 \mathrm{~mL}$ of water. surface; (ii) silver is released in water as a function of immersion time, reaching a stationary concentration after approximately 2-3 days; (iii) as already observed in similar systems, the most of dissolved silver is released in the first 24-48 hours of immersion. As we have already demonstrated, silver release should involve the formation or the thickening of $\mathrm{a}_{2} \mathrm{O}$ layer on the water-exposed surface of silver nano-objects, with the formation of an oxidized silver layer in the initial 1-2 days period, reaching a steady-state in which the slowly released $\mathrm{Ag}^{+}$ions are replaced by $\mathrm{Ag}$ oxidized from the bulk. ${ }^{5}$

As we start from a quite high surface concentration of silver, also the released amount is higher than the value which is usually released from monolayers made of small spherical silver nanoparticles grafted on glass. ${ }^{3,5-7}$ As can be easily calculated, anyway, the amount of silver released in these conditions after 10 days is less than $5 \%$ (about $2.5 \times 10^{-7} \mathrm{~g} \mathrm{~cm}^{-2}$ ) of the total concentration of silver present on the surface. This allows to imagine surfaces retaining for a long time the possibility to release silver ions in an environment subjected to flow of aqueous media, a feature that is considered essential for fight bacterial proliferation leading to severe infection and/or biofilm formation: for example, recently it was demonstrated that biofilm growing on a biomaterial surface is a dynamic process able to reach a maximal thickness at $12-18 \mathrm{~h}$ and maturation by 24 h. ${ }^{31}$ SEM images and UV-Vis-NIR spectra were taken for selected
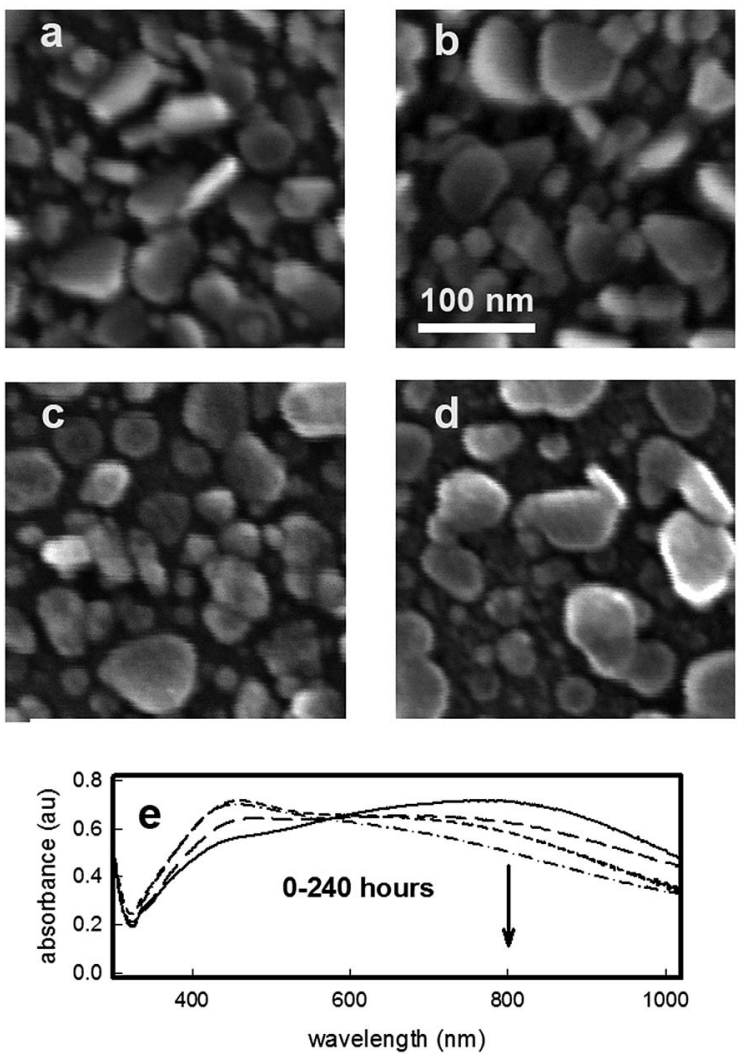

Fig. $4(a-d)$ SEM images of SURF-PEI-PLATES samples after increasing immersion times in pure water: (a) 0 hours (as prepared), (b) $5 \mathrm{~h}$ immersion, (c) $24 \mathrm{~h}$ immersion, (d) $240 \mathrm{~h}$ immersion; (e) UV-VisNIR spectra of the same samples. 
samples after the immersion in water and silver ion release. Fig. 4 shows the SEM images taken for samples after immersions of 5 (Fig. 4b), 24 (Fig. 4c) and 240 hours (Fig. 4d), compared with the untreated, as-prepared sample (Fig. 4a).

One can observe a progressive but limited consumption of the prismatic plates, causing a visible morphology change, from triangular like plates to more discoidal shapes. This of course modifies the UV-Vis-NIR spectra of the samples, causing a progressive depletion of the extinction at $800 \mathrm{~nm}$, as reported in Fig. 4e: the consumption of the plates results in a progressive flattening of the absorption in the NIR nm zone, as reduction of longitudinal dimensions as well the snipping of edges are expected to reduce the intensity and blue shift the dipolar inplane resonance. ${ }^{17}$ On the other side, as can be clearly seen from large area SEM images (see Fig. S8 in ESI $\dagger$ ), the overall homogeneity of the silver coating layer is preserved. Visually, the dark blue colour of the slides fades with prolonged immersion, as obvious consequence of the weakening of the broad band at $800 \mathrm{~nm}$. In the same time a more yellowish aspect can be noticed, depending on the increase of the band in the 450-500 nm range, typical of discoidal and spheroidal silver nano-objects. ${ }^{17}$ A photograph of a glass slide after 240 hours of immersion is reported in the ESI (see Fig. S9†): the dark blue colour has faded and a yellow nuance can be seen. Morphological changes caused by long immersion times in water can thus be perceived by the naked eye. This feature could also be exploited to assess the material shelf life and usability: the complete vanishing of the blue colour indicates the disappearing of the broad absorption at $800 \mathrm{~nm}$, and excludes the possibility of any exploiting of NIR laser excitation.

\section{Photo-thermal studies}

The photo-thermal behaviour of the samples obtained with different growth time has been measured by irradiation with a $808 \mathrm{~nm}$ continuous laser source $(200 \mathrm{~mW}$, spot diameter $1 \mathrm{~cm}$,
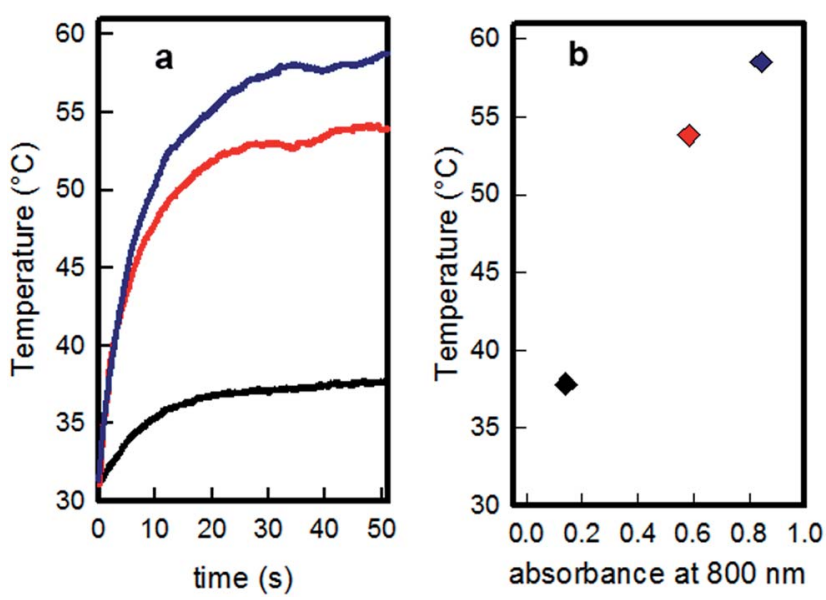

Fig. 5 (a) Temperature versus time thermograms obtained for samples under laser irradiation at $808 \mathrm{~nm}, 0.26 \mathrm{~W} \mathrm{~cm}^{-2}$ : samples grown 15 minutes (black line), 30 minutes (red line) and two hours SURF-PEI-PLATES (blue line); (b) maximum temperature reached by each sample vs. its absorbance at $808 \mathrm{~nm}$. resulting irradiance $0.26 \mathrm{~W} \mathrm{~cm}^{-2}$ ) on $10 \times 10 \mathrm{~cm}$ glass slides, which were obtained by cutting the larger slides typically used in the samples preparation. Thermograms (temperature vs. time graphs) were recorded using a thermocamera to register the temperatures reached by the glass samples while irradiated, observing in all cases an increase-plateau profile, reaching a stationary temperature within 40 seconds, as reported in Fig. 5a.

Temperature values recorded at plateau were of $37.8(0.3)$, $53.8(0.3)$ and $58.5(0.3)$ degrees for the samples grown 15 minutes, 30 minutes and two hours, respectively. As can be seen from Fig. $5 b$, the maximum temperature obtained by the three different samples increases with the absorbance value of each sample at $808 \mathrm{~nm}$, measured from spectra reported in Fig. 1, and the highest temperature is obtained when the laser source wavelength matches the $\lambda_{\max }$ of LSPR absorption. Uv-Vis-NIR spectra were recorded before and after irradiation for each sample, showing that the described treatment and relative produced hyperthermia did not change the LSPR features of the samples (an example is given in Fig. S10 in ESI†).

A similar set of experiments was repeated on a sample of SURF-PEI-PLATES (two hours growth) after 24 hours of immersion in water, to check if the silver release, producing the objects consumption and changes in LSPR features described in Fig. 4, could change drastically the photo-thermal effect. Only a slight decrease is observed in the value of temperature reached after irradiation of these samples (about $55{ }^{\circ} \mathrm{C}$, see Fig. S11 in ESI $\dagger$ for the thermogram), as it could be expected from the LSPR absorption at $808 \mathrm{~nm}$, which decreases only marginally after 24 hours of immersion in water as reported in Fig. 4e.

To our knowledge, there are no precise data about the temperatures needed to eliminate bacteria with photo-thermal effects. Moreover, temperature values measured by thermograms in the showed experiments describe temperature changes on bulk samples, a value which is a function of several experimental parameters, ${ }^{\mathbf{1 0}}$ giving no information on the temperatures changes in the close surroundings of the nanoobjects, which should reach considerably higher values. Anyway, it is known that heating of E. coli cells at temperatures of $52{ }^{\circ} \mathrm{C}$ for more than 5 minutes results in the destruction of bacteria permeability barrier. ${ }^{32}$ Recently it was reported that irradiating for 5 minutes with a laser a cluster of gold nanorods it is possible to reach a temperature of about $60^{\circ} \mathrm{C}$ causing the death of most bacterial cells in a $E$. coli biofilm. ${ }^{33}$ On the other side, we demonstrated that a few degrees of hyperthermia, caused by low irradiance laser excitation of a monolayer of GNSs on a glass bulk surface for longer times (30 $\mathrm{min})$, is sufficient to almost completely eliminate the bacteria cells of a $S$. aureus biofilm layer. ${ }^{11}$

\section{Antibacterial tests}

The antimicrobial activity of SURF-PEI-PLATES surfaces against $E$. coli and $S$. aureus was investigated. An established procedure allows the evaluation of the microbicidal effect (ME) in a thin liquid film in contact with the functionalized surface using the 
Table 1 ME values ${ }^{a}$ for SURF-PEI-PLATES samples

\begin{tabular}{llll}
\hline & $20 \mathrm{~min}$ & $5 \mathrm{~h}$ & $24 \mathrm{~h}$ \\
\hline E. coli & $<0.3$ & $0.9(0.3)$ & $>5$ \\
S. aureus & $<0.3$ & $0.8(0.3)$ & $>5$
\end{tabular}

${ }^{a}$ Values are obtained as the average of three measurements. $\mathrm{ME}=$ $\log N_{\mathrm{C}}-\log N_{\mathrm{E}}$. A value higher than 5 indicates that no survived bacteria were found after the test.

following formula: $\mathrm{ME}=\log N_{\mathrm{C}}-\log N_{\mathrm{E}}$, where $N_{\mathrm{C}}$ is the number of CFU $\mathrm{mL}^{-1}$ developed on the unmodified control glasses, and $N_{\mathrm{E}}$ the number of CFU $\mathrm{mL}^{-1}$ counted after exposure to modified glasses $\left(\mathrm{CFU}=\right.$ colony forming unit). ${ }^{5}$

Experimental results after contact times of 20 minutes, 5 and $24 \mathrm{~h}$ are reported in Table 1, and show that SURF-PEI-PLATES glass slides display a significant bactericidal effect after a long contact time. 24 hours contact times are able to exert a complete microbicidal effect, eliminating at least $99.999 \%$ of bacteria. $\$$ The effect is much less intense with 5 hours of contact, while no microbicidal effect is present with very short contact times: it must be noted that silver release, which is considered as the main responsible of antibacterial action, is limited $(0.015 \times$ $10^{-6} \mathrm{~g} \mathrm{~cm}^{-2}$, see Fig. 3) after a 20 min time of contact with water. Mechanisms by which AgNPs exert their antibacterial effect are still hugely debated, anyway Xiu $e t$ al. ${ }^{34}$ showed that silver nano-objects are devoid of antibacterial action when used under anaerobic conditions which exclude oxidation to silver( $\mathrm{I}$ ), thus preventing its release. SURF-PEI-PLATES samples action against $S$. aureus for long contact times seems much more intense than the one found for SURF-PEI-SEEDS samples bearing small spherical AgNPs, ${ }^{6}$ and this should be due to the higher concentration of silver present on the former. Anyway, at this preliminary stage of investigation, one cannot exclude that also the different morphology of objects on surfaces is playing a complex role. Remarkably, in the tests proposed by CEN (European Committee for Standardization) in - EN 13697, $\$$ the microbicidal activity of a disinfectant is considered acceptable when the decimal-log reduction rate (i.e. ME), is at least equal to 4 after 5 min of contact. In our case ME of the modified glass is superior to 4 after $24 \mathrm{~h}$ of contact for both strains, with a long term action which seems well suited to build antibacterial surfaces for medical devices.

Surfaces functionalization with silver nano-objects able to release controlled quantities of $\mathrm{Ag}(\mathrm{I})$ and imparting antibacterial features, is a technique that was widely investigated in the last decade. So, these first set of results was largely expected, on

\footnotetext{
$\$$ As the CFU counted in the control measures reach at least a value of $10^{5}$, although varying every time as consequence of differences in the experimental conditions, when no $\mathrm{CFU}$ can be found in the samples coming from SURF-PEI-PLATES we assign to ME a value of 5 , to indicate that at least 99.999\% of bacteria were eliminated during the test.

$\S$ European Committee for Standardization, EN 13697, Chemical Disinfectants and Antiseptics - Quantitative Non-porous Surface Test for the Evaluation of Bactericidal and/or Fungicidal Activity of Chemical Disinfectants used in Food, Industrial, Domestic and Institutional Areas - Test Method and Requirements (Phase 2, Step 2), Brussells: CEN, 2002.
}

Table $2 \quad M_{\mathrm{T}}$ values $^{a}$ for SURF-PEI-PLATES samples

\begin{tabular}{lll}
\hline & $20 \mathrm{~min}$ & $\begin{array}{l}20 \mathrm{~min} \\
\text { (after } 24 \mathrm{~h} \text { in water) }\end{array}$ \\
\hline E. coli & $>5$ & $>5$ \\
S. aureus & $3.7(0.3)$ & $3.8(0.3)$
\end{tabular}

${ }^{a}$ Values are obtained as the average of three measurements. $\mathrm{ME}_{\mathrm{T}}=$ $\log N_{\mathrm{C}}-\log N_{\mathrm{T}}$. A value higher than 5 indicates that no survived bacteria were found after the test.

the basis of several reported studies..$^{3-7,35-37}$ Anyway, in all the studies related to antibacterial effects based on silver nanoobjects, hyperthermia caused by photo-thermal effects was never considered as an option to enhance their antimicrobial activity when grafted on surfaces. On the other side, hyperthermia based antimicrobial applications relies usually on magnetic and/or gold nanoparticles. The result is that no examples are reported about antimicrobial action caused by photo-thermal effects given by silver nanoplates, nor in colloidal suspension neither grafted to a surface. Our main interest, thus, was to obtain a novel and (until now) undescribed, antimicrobial action given by NIR laser excitation of anisotropic silver nano-objects. The final goal was to have an hyperthermia induced action which could be "switched on" as a result of NIR laser irradiation, in addition to the expected long term action due to silver release usually exhibited by silver AgNPs layers.

To obtain this, SURF-PEI-PLATES samples, opportunely cut in order to be completely covered by the light spot, were irradiated for $20 \mathrm{~min}$ with a $808 \mathrm{~nm}$ laser source, while in contact with the described bacteria suspensions, using an irradiance of $0.26 \mathrm{~W} \mathrm{~cm}^{-2}$. In this case the "thermal microbicidal effect", $\mathrm{ME}_{\mathrm{T}}$ was calculated with the following formula:

$$
\mathrm{ME}_{\mathrm{T}}=\log N_{\mathrm{C}}-\log N_{\mathrm{T}}
$$

where $N_{\mathrm{C}}$ is the number of $\mathrm{CFU} \mathrm{mL}^{-1}$ developed after the contact with SURF-PEI-PLATES samples in absence of irradiation, taken as control, and $N_{\mathrm{T}}$ the number of $\mathrm{CFU} \mathrm{mL}{ }^{-1}$ counted after exposure to SURF-PEI-PLATES sample in presence of irradiation.

It is important to stress that in these conditions $\mathrm{ME}_{\mathrm{T}}$ has to be ascribed only to the photo-thermal effect, as any possible influence from the limited silver release in absence of irradiation is considered in the control count. Moreover, as very limited silver ion release is observed after 20 minutes (see Fig. 3), no silver related ME is observed during this time (see Table 1). It is also important to stress that the $\mathrm{ME}_{\mathrm{T}}$ values do not change when unmodified, blank glass samples (both irradiated and not irradiated) are used to obtain control $\mathrm{CFU}$ values instead of not irradiated, functionalized samples. This clearly states that laser irradiation alone, in absence of plasmonic objects exerting photo thermal features, does not cause any harm to bacteria.

As reported in Table 2, in the case of $\mathrm{ME}_{\mathrm{T}}$ we observe an higher activity against $E$. coli than against $S$. aureus. This may be 
due to the fact that Gram positive bacteria present a relatively thick (20-80 nm) and continuous cell wall consisting mainly of peptidoglycane, while Gram negative bacteria feature a thinner peptidoglycane layer $(5-10 \mathrm{~nm})$ surrounded by an outer phospholipidic membrane, which is expected to be less solid and resistant. The $\mathrm{ME}_{\mathrm{T}}$ test result show that the laser treatment kills more than $99.999 \%$ of $E$. coli and about $99.95 \%$ of $S$. aureus cells in 20 minutes of irradiation at $0.26 \mathrm{~W} \mathrm{~cm}^{-2}$, a value which is below the maximum allowed for exposure of the $\operatorname{skin}^{38}$ and using a wavelength which is suitable for in vivo use.

At this point, one question arising was whether antibacterial effect given by laser irradiation was due only to hyperthermia or mediated by an augmented release of silver ions due the hyperthermia itself, as is reported for some described systems. ${ }^{\mathbf{1 2 - 1 4}}$ Thus, we measured the quantity of silver released in a fixed volume $(3 \mathrm{~mL})$ of water after 20 minutes of irradiation in the same conditions used for the $\mathrm{ME}_{\mathrm{T}}$ tests (laser spot $1 \mathrm{~cm}^{2}$, irradiance $0.26 \mathrm{~W} \mathrm{~cm}^{-2}$ ). After irradiation, the solution was brought to ICP, revealing that the quantity of silver ion released from the surface after 20 minutes of laser irradiation was identical, in the precision limits of the techniques, to the one found after 20 minutes of immersion in water in absence of irradiation $\left(0.015 \times 10^{-6} \mathrm{~g} \mathrm{~cm}^{-2}\right)$. Thus, we can state that the laser irradiation produces an antibacterial effect apparently due to hyperthermia only, and is not connected to an augmented silver release.

But what about the possibility to user the laser irradiation on SURF-PEI-PLATES samples after their immersion in water, to simulate a situation in which, in addition to a long-term microbicidal activity imparted by silver ions release, a reinforce of antibacterial action was desired? To answer this question, we repeated the $\mathrm{ME}_{\mathrm{T}}$ tests on SURF-PEI-PLATES samples after their immersion in $3 \mathrm{~mL}$ of water for 24 hours. As we have already described, thermograms did not show dramatic decrease of photo-thermal activity after immersion in water for $24 \mathrm{~h}$, as UV-Vis-NIR spectra still show an intense absorption at $808 \mathrm{~nm}$. As can be seen in Table 2, the $\mathrm{ME}_{\mathrm{T}}$ in these conditions is completely conserved, suggesting once again that the antibacterial action obtained within the 20 minutes irradiation is caused by hyperthermia from the silver nano-objects: as long as they absorb sufficient radiation at the proper wavelength, hyperthermia is produced and antibacterial action takes place.

\section{Conclusions}

We demonstrated, for the first time, the coating of bulk surfaces with silver nanoplates which can exert an antibacterial action based on the photo-thermal effect, which can be switched on by a proper laser excitation. This novel kind of antimicrobial action is added to the well-known microbicidal abilities typically showed by AgNPs containing surfaces. The goal was reached by tuning the LSPR features of the nanoplates using a new seed growth synthesis, operated directly on bulk surfaces in absence of any surfactant or other harmful chemicals. We started by grafting small silver nanoparticles on bulk glass, previously treated with PEI-silane as grafting agent. This allowed to prepare stable (showing no release of nano-objects in the environment) and reproducible samples bearing an homogeneous layer of flat silver nano-objects of various dimensions and shapes, with an overall broad absorption spectra having a maximum centered around $800 \mathrm{~nm}$. Under laser irradiation at $808 \mathrm{~nm}$ and at safe irradiance values, in 20 minutes the almost complete elimination of bacteria $S$. aureus and $E$. coli is obtained in vitro through laser-induced hyperthermia. Of course, a complete microbicidal action is observed also in longer timescales, as a result of the contact with the surface, an expected $^{3-7}$ long-term antibacterial action which is explained with the slow and controlled release of silver ions from the nanoobjects. Long time immersions in water produce morphological changes of the grafted object, reasonably related to silver ions release, but a 24 hours immersion do not affect seriously the antibacterial action obtainable by laser induced hyperthermia. We believe this new approach will have promising applications to several types of surfaces (prosthetic and subcutaneous devices, surgical sutures, wound dressings, to name a few examples), as it offers a long term antimicrobial protection which can be reinforced, on need, with the laser induced action.

\section{Acknowledgements}

We gratefully acknoledge Prof. M. P. Riccardi, Arvedi Laboratory, CISRiC, Pavia, for carrying out SEM experiments. We also thank Prof. Marcella Bini, Department of Chemistry, University of Pavia, for XRD measures and fruitful discussions.

\section{Notes and references}

1 D. Campoccia, L. Montanaro and C. R. Arciola, Biomaterials, 2013, 34, 8018.

2 P. Pallavicini, G. Dacarro, Y. A. Diaz-Fernandez and A. Taglietti, Coord. Chem. Rev., 2014, 275, 38.

3 A. Taglietti, Y. A. Fernandez, E. Amato, L. Cucca, G. Dacarro, P. Grisoli, V. Necchi, P. Pallavicini, L. Pasotti and M. Patrini, Langmuir, 2012, 28, 8140.

4 E. Amato, Y. A. Diaz-Fernandez, A. Taglietti, P. Pallavicini, L. Pasotti, L. Cucca, C. Milanese, P. Grisoli, C. Dacarro, J. M. Fernandez-Hechavarria and V. Necchi, Langmuir, 2011, 27, 9165.

5 P. Pallavicini, A. Taglietti, G. Dacarro, Y. A. Diaz-Fernandez, M. Galli, P. Grisoli, M. Patrini, G. Santucci De Magistris and R. Zanoni, J. Colloid Interface Sci., 2010, 350, 110.

6 G. Dacarro, L. Cucca, P. Grisoli, P. Pallavicini, M. Patrini and A. Taglietti, Dalton Trans., 2012, 41, 2456.

7 A. Taglietti, C. R. Arciola, A. D'Agostino, G. Dacarro, L. Montanaro, D. Campoccia, L. Cucca, M. Vercellino, A. Poggi, P. Pallavicini and L. Visai, Biomaterials, 2014, 35, 1779.

8 D. Jimenez de Aberasturi, A. B. Serrano-Montesadv and L. M. Liz-Marzán, Adv. Opt. Mater., 2015, 3, 602.

9 W. Jo and M. J. Kim, Nanotechnology, 2013, 24, 195104.

10 S. A. Khan, A. K. Singh, D. Senapati, Z. Fan and P. C. Ray, J. Mater. Chem., 2011, 21, 17705. 
11 P. Pallavicini, A. Donà, A. Taglietti, P. Minzioni, M. Patrini, G. Dacarro, G. Chirico, L. Sironi, N. Bloise, L. Visai and L. Scarabelli, Chem. Commun., 2014, 50, 1969.

12 S. Mo, X. Chen, M. Chen, C. He, Y. Lu and N. Zheng, J. Mater. Chem. B, 2015, 3, 6255.

13 C. Fasciani, M. J. Silvero, M. A. Anghel, G. A. Arguello, M. C. Becerra and J. C. Scaiano, J. Am. Chem. Soc., 2014, 136, 17394.

14 K. C. L. Black, T. S. Sileika, J. Yi, R. Zhang, J. G. Rivera and P. B. Messersmith, Small, 2014, 10, 169.

15 S. Boca-Farcau, M. Potara, T. Simon, A. Juhem, P. Baldeck and S. Astilean, Mol. Pharm., 2014, 11, 391.

16 S. C. Boca, M. Potara, A. M. Gabudean, A. Juhem, P. L. Baldeck and S. Astilean, Cancer Lett., 2011, 311, 131.

17 I. Pastoriza-Santos and L. M. Liz-Marzan, J. Mater. Chem., 2008, 18, 1725.

18 A. Casu, E. Cabrini, A. Donà, A. Falqui, Y. Diaz-Fernandez, C. Milanese, A. Taglietti and P. Pallavicini, Chem.-Eur. J., 2012, 18, 9381.

19 M. A. Mahmoud, C. E. Tabor, M. A. El-Sayed, Y. Ding and Z. L. Wang, J. Am. Chem. Soc., 2008, 130, 4590.

20 Y. Sun, Chem. Mater., 2007, 19, 5845.

21 A. M. Alkilany, P. K. Nagaria, C. R. Hexel, T. J. Shaw, C. J. Murphy and M. D. Wyatt, Small, 2009, 5, 701.

22 K. H. Lee, K. M. Huang, W. L. Tseng, T. C. Chiu, Y. W. Lin and H. T. Chang, Langmuir, 2007, 23, 1435.

23 K. Aslan, Z. Leonenko, J. R. Lakowicz and C. D. Geddes, J. Phys. Chem. B, 2005, 109, 3157.

24 A. N. Sidorov, G. W. Sławinski, A. H. Jayatissa, F. P. Zamborini and G. U. Sumanasekera, Carbon, 2012, 50, 699.
25 K. Nouneh, M. Oyama, R. Diaz, M. A. Lefdil, I. V. Kityk and M. Bousmina, J. Alloys Compd., 2011, 509, 2631.

26 H. Jia, J. Zeng, J. An, W. Song, W. Xu and B. Zhao, Thin Solid Films, 2008, 516, 5004.

27 Y. K. Kim and D. H. Min, $R S C A d v .$, 2014, 4, 6950.

28 D. Aherne, D. M. Ledwith, M. Gara and J. M. Kelly, Adv. Funct. Mater., 2008, 18, 2005.

29 D. M. Ledwith, A. M. Whelan and J. M. Kelly, J. Mater. Chem., 2007, 17, 2459.

30 J. Liu, D. A. Sonshine, S. Shervan and R. H. Hurt, ACS Nano, 2010, 4, 6903.

31 G. Q. Zhao, L. H. Ye, Y. C. Huang, D. K. Yang, L. Li and G. Xu, Cell Biochem. Biophys., 2011, 61, 371.

32 N. Katsui, T. Tsuchido, R. Hiramatsu, S. Fujikawa, M. Takano and I. Shibasaki, J. Bacteriol., 1982, 151, 15231531.

33 W. Jo and M. J. Kim, Nanotechnology, 2013, 24, 195104195111.

34 Z. M. Xiu, Q. B. Zhang, H. L. Puppala, V. L. Colvin and P. J. Alvarez, Nano Lett., 2012, 12, 4271.

35 J. Chen, S. Li, J. Luo, R. Wan and W. Ding, J. Nanomater., 2016, 7135852.

36 D. Paul, S. Paul, N. Roohpour, M. Wilks and P. Vadgama, J. Funct. Biomater., 2013, 4, 358-375.

37 H. Zhang, W. Meng and S. Ayusman, Silver nanoparticle antimicrobials and related materials, in Nanoantimicrobials, Springer, Berlin, Heidelberg, 2012, pp. 3-45.

38 ANSI, American National Standard for Safe Use of Lasers, Laser Institute of America, Orlando, FL, 2000. 